\title{
Predicting nonpolymeric materials structure with real-space self-consistent field theory
}

\author{
R. B. Thompson \\ Department of Physics, University of Waterloo, Waterloo, Ontario, Canada N2L $3 G 1$ \\ (Received 21 July 2005; revised manuscript received 2 November 2005; published 17 February 2006)
}

\begin{abstract}
Polymer self-consistent field theory of the Edwards-Helfand kind is the state-of-the-art method for predicting the morphologies of block copolymer materials. The methodology of block copolymer self-consistent field theory is transported to classical density functional theory such that a wide range of self-consistent field theory tools can be applied to completely nonpolymeric materials, such as liquid crystal, molecular, or colloidal systems. This allows for the prediction of structure in nonpolymeric condensed matter systems without any prior knowledge of the possible phases, using calculations that take a fraction of the time needed for simulations. The approach is applied to a simple interaction site density functional theory representing adsorbed nitrogen molecules, and plastic crystal as well as herringbone phases are found in the phase diagram.
\end{abstract}

DOI: 10.1103/PhysRevE.73.020502

PACS number(s): 61.30.Cz, 81.30.Dz, 82.70.Dd, 89.75.Fb

The properties of new materials and hence their utility is fundamentally connected with their structures. The prediction of material structures is therefore of central importance in condensed matter physics and materials science. Polymer self-consistent field theory (SCFT) of the Edwards-Helfand kind is at the forefront of predictive methodologies for the structures of block copolymer materials due to its computational efficiency and accuracy, and the lack of any need for $a$ priori assumptions about the possible structures. Here it is described how SCFT can be used to predict the morphologies of completely nonpolymeric materials with the same efficiency and robustness as for block copolymers.

Liquid crystals and molecular or colloidal particles are often described with classical density functional theory (DFT) [1-3], and it is by combining aspects of DFT with SCFT that the structures of these systems can be obtained. This combination is natural since both theories describe systems in terms of a free energy functional of a density field. In the past, there have been two main obstacles in applying DFT to general problems: (i) Due to the orientational independence, research has focused more on spherical systems. (ii) The density field is typically parametrized to have the symmetry of the various phases being investigated. Therefore the phases must be presupposed.

The first restriction has been largely overcome. There are two standard approaches. One is to directly write a freeenergy functional for anisotropic particles. This can be very challenging, but a number of researchers have attempted it. See for example Refs. [4-6]. The other is to use an interaction site model DFT, as suggested by Chandler, Singer, and McCoy $[7,8]$. This is an extremely versatile and general approach, to be preferred in many situations. The second restriction is more severe. It is the purpose of this paper to present a solution to this second problem based on recent advances in SCFT $[9,10]$.

SCFT is a mean-field, equilibrium, statistical mechanical theory that is well known as a state-of-the-art approach for solving problems in polymeric, and particularly, block copolymer systems [11]. It is normally solved numerically, and the earliest solutions were achieved through an iteration approach in one dimension only, much as is commonly done in DFT. A powerful spectral approach was later introduced
[12] that allowed SCFT to be solved accurately and efficiently in higher dimensions. This is analogous to parametrization approaches [13] (often Gaussians, for example) in DFT. Most recently, a method for solving SCFT in higher dimensions with no a priori knowledge of the possible morphologies was introduced that uses false dynamics on chemical potential fields $[14,15]$. This method has since been enhanced by powerful Fourier transform techniques $[16,17]$, convergence improvements $[18,19]$, interpolation methods [18], and variable cell methods [20], resulting in a suite of tools that puts it at the forefront of materials science predictive methodologies, at least for polymeric systems. These last SCFT developments have not been mirrored in DFT. It would be extremely valuable to transfer these SCFT advances to DFT so that nonpolymeric materials could be studied with similar predictive power. As the SCFT and DFT formalisms are entirely compatible, such a transfer would seem most natural. Indeed, DFT and SCFT have been used together in other polymer-type applications in the past [21-24]. Here, all polymer aspects are removed and a DFT theory based on SCFT methodology is examined for liquid crystal, colloidal, or molecular systems. The method is adaptable to a wide variety of existing DFTs.

Given a Hamiltonian, the free-energy functional for an arbitrary system can be derived through the standard SCFT approach, which is based on a continuous delta function substitution in a particle-based partition function in order to get a field-based representation; this is described in depth in Ref. [15]. If the system has hard core interactions, such as one might expect in molecular, colloidal, or liquid crystal systems, the hard core potential term can be carried through from the Hamiltonian without being specified, resulting in an unspecified free-energy term that can be approximated in any number of DFT ways. This is a different approach from standard DFT derivations but results in the same free-energy expressions. For concreteness, consider a system of hard rods comprised of either tangent or fused hard spheres of diameter $\sigma$. The dimensionless free energy per volume $V$ of this system would be 


$$
\begin{aligned}
\frac{F \sigma^{3}}{k_{B} T V}= & \frac{\sigma^{3}}{V}\left\{\frac{\rho_{\mathrm{s}} V}{N}\left[\ln \left(\frac{\rho_{\mathrm{s}} \sigma^{3}}{N}\right)-\ln \left(\frac{Q}{4 \pi V}\right)\right]-\int d \mathbf{r} w(\mathbf{r}) \rho(\mathbf{r})\right. \\
& \left.+\frac{1}{2} \int d \mathbf{r} d \mathbf{r}^{\prime} \rho(\mathbf{r}) \phi_{\mathrm{att}}\left(\left|\mathbf{r}-\mathbf{r}^{\prime}\right|\right) \rho\left(\mathbf{r}^{\prime}\right)+F_{\mathrm{ex}}[\rho]\right\},
\end{aligned}
$$

where $k_{B}$ is Boltzmann's constant and $T$ is the temperature. Further, $N$ is the number of spheres in each rod, $\rho_{s}$ is the overall number density of spheres, and $\rho(\mathbf{r})$ is the local number density of spheres. $Q$ is given by

$$
Q=\int d \mathbf{u} d \mathbf{r} \exp \left\{-\sum_{\alpha=1}^{N} w[\mathbf{r}+(\alpha-1) l \mathbf{u}]\right\}
$$

and is the partition function of a single-rod subject to the dimensionless chemical potential field $w(\mathbf{r})$, where this $w(\mathbf{r})$ field determines the distribution of spheres $\rho(\mathbf{r})$. Likewise, the distribution of spheres $\rho(\mathbf{r})$ determines the distribution of the chemical potential field $w(\mathbf{r})$. The system of mean-field equations relating $\rho(\mathbf{r})$ and $w(\mathbf{r})$ is obtained by varying (1) with respect to $\rho(\mathbf{r})$ and $w(\mathbf{r})$, and these equations are then solved for these two fields self-consistently. The vector $\mathbf{u}$ in (2) represents orientational dependences, and $l$ is the distance between spherical centers in the rod. The fourth term on the right-hand side of (1) is an attractive mean-field term based on a two-body Lennard-Jones potential $\phi_{\text {att }}$; the strength of this potential is adjusted through a parameter $\varepsilon$, and the minimum of the potential falls at a separation $r_{\min }=2^{1 / 6} \sigma$. Only the attractive part of the potential is used following the Weeks, Chandler, Anderson approach [25] (see also references in Ref. [10]). The last term $F_{\text {ex }}$ on the right-hand side is the excess (beyond ideal gas) free energy and represents the hard-sphere repulsion. In the following, for clarity, one of the simplest approximations that gives good qualitative results is chosen, however many different DFT terms could be used in principle, and so the methodology of the SCFT approach is general enough to admit quantitative results. The weighted density approximation (WDA) of Tarazona [26] is used here since (1) can be reduced to the interaction site model of Talanquer and Oxtoby [27] for hard dumbbells when written in the grand canonical SCFT formalism [28,29] while ignoring orientational dependences and setting $N=2$. Other approximations are also possible [30].

While stressing that almost any liquid crystalline, molecular, or colloidal shape may be represented with generalizations of the above formalism, it is instructive to show how SCFT methods can predict the crystal structures of systems without any a priori knowledge through a concrete example. To this end, let the system be simplified further to examine rods comprising only two spheres each. Furthermore, although (1) is written in three dimensions, let a twodimensional (2D) system be examined for clarity [31]. Such 2D homonuclear hard dumbbells have been studied in the past with Monte Carlo simulations and free volume methods [32], although only for near tangent sphere systems. Here, one would like to start with a small anisotropy-although this is by no means required-and so the center-center separation of the spheres is taken to be $0.33 \sigma$ using the method of Cherepanova and Stekolnikov [33]. This system then roughly

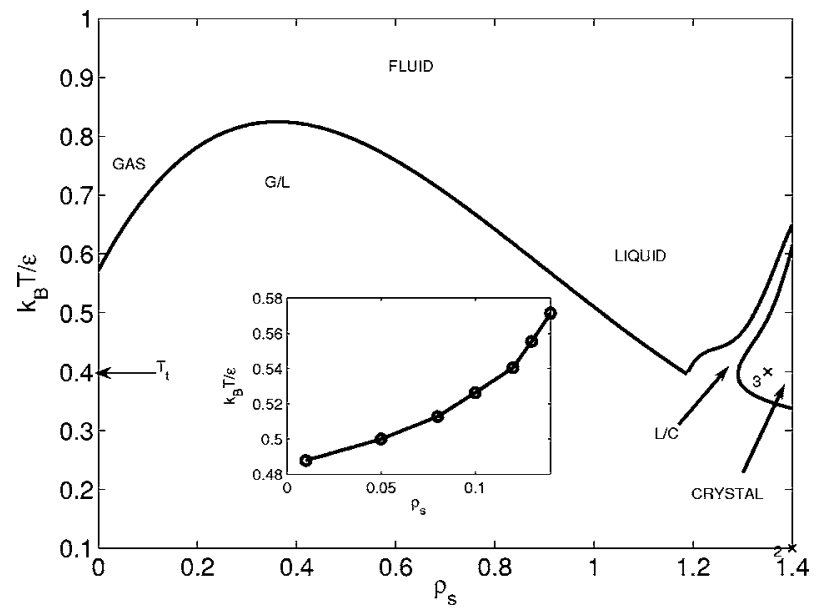

FIG. 1. Phase diagram for a $2 \mathrm{D} \mathrm{N}_{2}$ system. $\varepsilon$ is a parameter setting the strength of the Lennard-Jones interaction. Phase boundaries have been smoothed to remove numerical noise. $G / L$ denotes gas-liquid coexistence, and $L / C$ denotes liquid-crystal coexistence. The crosses denoted with " 2 " and " 3 " indicate the locations of the morphologies depicted in Figs. 2 and 3, respectively. The arrow indicates the anticipated triple point. The inset shows the spinodal line at low densities.

corresponds to nitrogen $\mathrm{N}_{2}$ molecules adsorbed on a surface [34].

Using random initial fields $w(\mathbf{r})$, the self-consistent equations were solved using false-dynamics and Fourier methods, together with a variation on the Anderson mixing technique described in Ref. [19]. The resultant fields $w(\mathbf{r})$ and $\rho(\mathbf{r})$ were substituted back into the two-dimensional version of the free-energy expression (1), which was then minimized with respect to the calculation box size using a simplex method to give a commensurate periodic structure [35,36]. In some cases, greater accuracy was required for the free energies, and so a Fourier space interpolation scheme similar to that described in Ref. [18] was used to increase the morphological resolution. The optimized free energies, together with double-tangent constructions, allowed for the mapping of a phase diagram for the 2D $\mathrm{N}_{2}$ system, displayed in Fig. 1. It shows the gas, liquid, and solid phases of the system for nearly all overall sphere number densities [37] and a wide temperature range. The qualitative phases found are consistent with the expected phases for similar systems studied in both two and three dimensions using other approaches (see, for example, Refs. $[32,34,38]$ ). A triple point is marked at a dimensionless temperature $k_{B} T / \varepsilon=0.4$; although on the scale of the diagram the left boundary of the coexistence region stops slightly below 0.6 , the inset shows the spinodal line that extends much lower in temperature. The binodal line, although it could not be distiguished within the present accuracy of this work, should exist to the left (lower density side) of the spinodal line, and it is anticipated that the gas phase will continue to exist at lower temperatures but with a phase line that is very close to a zero density value along most of the left side of the diagram. At low temperatures, the crystal phase forms roughly a herringbone pattern. An example of this is shown in Fig. 2, which is for a number density of $\rho_{s} \sigma^{2}=1.4$ and a reduced temperature of 0.1 . A 


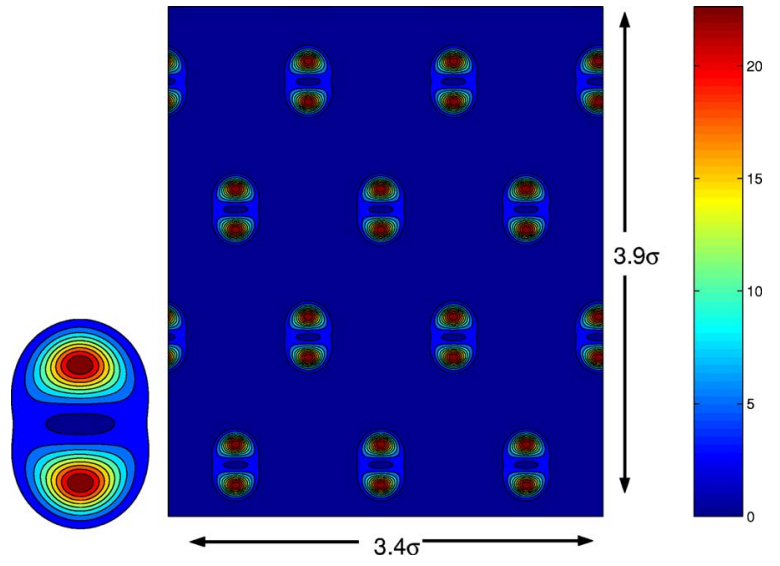

FIG. 2. (Color online) Average density distribution $\sigma^{2} \rho(\mathbf{r})$ for the $2 \mathrm{D} \mathrm{N}_{2}$ system at $\rho_{s} \sigma^{2}=1.4$ and $k_{B} T / \varepsilon=0.1$. In the lower left corner a blowup of the distribution in the vicinity of one localized molecule is shown.

blowup of the sphere-number density distribution for a specific molecule is shown in the lower left of Fig. 2. The herringbone pattern forms spontaneously in the substrate-free system, showing that it is very robust at low temperatures. At lower densities or higher temperatures, a plastic crystal phase is found, where the center of mass of the $\mathrm{N}_{2}$ molecules are well localized, but the orientations are random. This is shown in Fig. 3 for $\rho_{s} \sigma^{2}=1.35$ and $k_{B} T / \varepsilon=0.4$. Note the lack of orientational ordering for the individual localized molecules compared with Fig. 2. The herringbone versus plasticcrystal structures are more apparent if one looks at the angular distribution of the sphere-number densities shown in Fig. 4. The solid line is the herringbone structure, whereas the dashed line is the plastic crystal orientational distribution. Note that the latter is very uniform compared to the herringbone. The inset is a blowup of the plastic-crystal distribution,

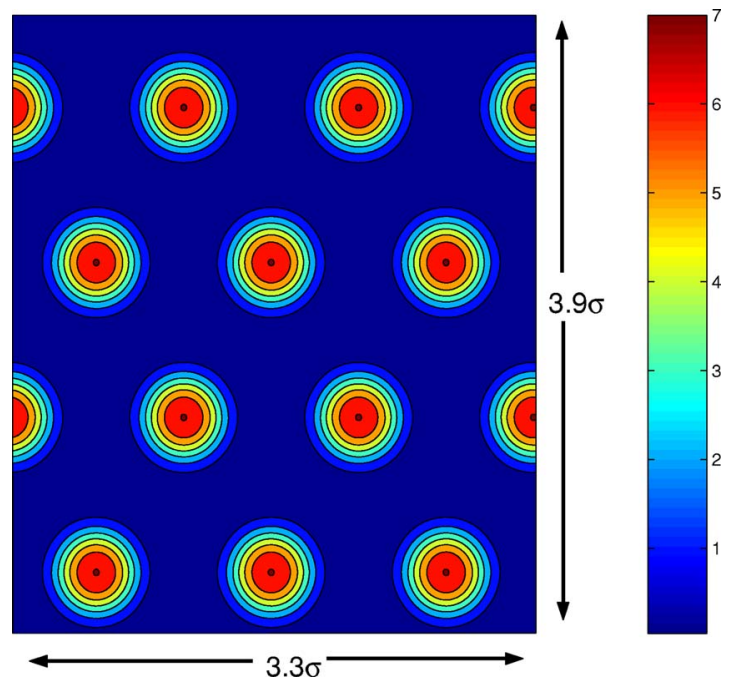

FIG. 3. (Color online) Average density distribution $\sigma^{2} \rho(\mathbf{r})$ for the $2 \mathrm{D} \mathrm{N}_{2}$ system at $\rho_{s} \sigma^{2}=1.35$ and $k_{B} T / \varepsilon=0.4$.

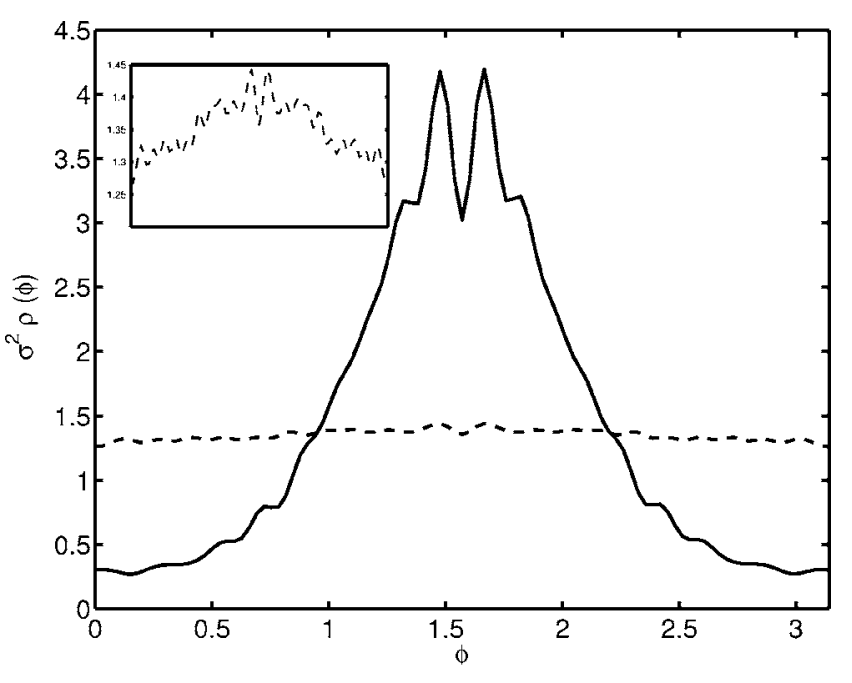

FIG. 4. Density distribution as a function of angle $\phi$ (radians) for the $2 \mathrm{D} \mathrm{N}_{2}$ system. The solid line depicts the herringbone phase at $\rho_{s} \sigma^{2}=1.4$ and $k_{B} T / \varepsilon=0.1$, while the dashed line is for the plastic crystal phase at $\rho_{s} \sigma^{2}=1.35$ and $k_{B} T / \varepsilon=0.4$. The inset shows a blowup of the plastic-crystal density distribution as a function of angle. The data has been processed to remove numerical noise.

showing that there is, on another scale, a structure very similar to the herringbone distribution. Indeed, no first-order transition was found between these two structures; it is possible that the transition is just too weak to be observed within present accuracy. On the other hand, the orientational distribution never loses all herringbone structure even in the seeming plastic-crystal phase as shown in the inset of Fig. 4. This implies that a corresponding orientational order parameter would never reach zero, thus preventing the delineation of a possible second-order transition. Instead, the herringbone and plastic-crystal phases seem to be most likely different extremes of a single phase in two dimensions, at least for this particular DFT approximation. It is likely, the simplistic nature of the weight function of the present DFT approach prevents the appearance of the first-order transition that is observed in 3D simulations [34]. DFT calculations in three dimensions do not predict any stable plastic-crystal phase at all for molecules with the present degree of anisotropy [38]; the present results are not inconsistent with this previous finding since the plastic-crystal phase here is most likely not a separate phase from the ordered phase, as previously discussed. It may be that the $2 \mathrm{D}$ system does actually behave differently than the threedimensional system.

A simple 2D system with small-shape anisotropy has been examined with a basic DFT functional in order to illustrate the ease with which whole-phase diagrams can be computed in a fraction of the time required for most simulations [39]. The SCFT methodology is independent of this simple system or DFT functional, and can be applied to any number of materials in order to predict the crystalline morphology without any prior knowledge of the possible phases. A whole suite of SCFT tools can in this way be transferred to the 
study of nonpolymeric materials, allowing materials structure to be computationally anticipated with efficiency, robustness, and, if more advanced DFTs are used, quantitative accuracy.

\section{ACKNOWLEDGMENTS}

This work was supported by the National Sciences and Engineering Research Council (NSERC) of Canada. A major part of the calculations were performed using SHARCNET .
[1] R. Evans, in Fundamentals of Inhomogeneous Fluids, edited by D. Henderson (Marcel Dekker, New York, 1992).

[2] G. J. Vroege and H. N. W. Lekkerkerker, Rep. Prog. Phys. 55, 1241 (1992).

[3] E. Tareyeva and V. Ryzhov, e-print cond-mat/9712265.

[4] E. Velasco, L. Mederos, and D. E. Sullivan, Phys. Rev. E 66, 021708 (2002).

[5] Z. Y. Chen, Phys. Rev. E 47, 3765 (1993).

[6] A. M. Somoza and P. Tarazona, J. Chem. Phys. 91, 517 (1989).

[7] D. Chandler, J. D. McCoy, and S. J. Singer, J. Chem. Phys. 85, 5971 (1986).

[8] See also references and discussion by M. B. Sweatman, J. Commun. Technol. Electron. 15, 3875 (2003).

[9] For non-SCFT methods of examining systems without presupposed phases, see Y. Bohbot-Raviv and Z.-G. Wang, Phys. Rev. Lett. 85, 3428 (2000); M. Valera, R. F. Bielby, and F. J. Pinski, J. Chem. Phys. 115, 5213 (2001); D. Gottwald, G. Kahl, and C. N. Likos, ibid. 122, 204503 (2005).

[10] Interaction site model DFT has been extensively applied by Frischknecht and co-workers to polymeric and related materials. See, for example, A. L. Frischknecht, J. D. Weinhold, A. G. Salinger, J. G. Curro, L. J. D. Frink and J. D. McCoy, J. Chem. Phys. 117, 10385 (2002); A. L. Frischknecht, J. G. Curro, and L. J. D. Frink, ibid. 117, 10398 (2002).

[11] M. W. Matsen, J. Commun. Technol. Electron. 14, R21 (2002), and references therein.

[12] M. W. Matsen, in Soft Matter, edited by G. Gompper and M. Schick (Wiley-VCH, Weinheim, 2005), Vol. 1.

[13] H.-J. Woo and P. A. Monson, J. Chem. Phys. 118, 7005 (2003).

[14] F. Drolet and G. H. Fredrickson, Phys. Rev. Lett. 83, 4317 (1999).

[15] G. H. Fredrickson, V. Ganesan, and F. Drolet, Macromolecules 35, 16 (2002).

[16] G. Tzeremes, K. Ø. Rasmussen, T. Lookman, and A. Saxena, Phys. Rev. E 65, 041806 (2002).

[17] K. Ø. Rasmussen and G. Kalosakas, J. Polym. Sci., Part B: Polym. Phys. 40, 1777 (2002).

[18] H. D. Ceniceros and G. H. Fredrickson, Multiscale Model. Simul. 2, 452 (2004).

[19] R. B. Thompson, K. Ø. Rasmussen, and T. Lookman, J. Chem. Phys. 120, 31 (2004).

[20] J. L. Barrat, G. H. Fredrickson, and S. W. Sides, J. Phys. Chem. B 109, 6694 (2005).

[21] R. B. Thompson, V. V. Ginzburg, M. W. Matsen, and A. C. Balazs, Science 292, 2469 (2001).

[22] F. Schmid, J. Phys.: Condens. Matter 10, 8105 (1998).

[23] F. Schmid, J. Chem. Phys. 104, 9191 (1996).
[24] M. Müller, L. G. MacDowell, and A. Yethiraj, J. Chem. Phys. 118, 2929 (2003).

[25] J. D. Weeks, D. Chandler, and H. C. Anderson, J. Chem. Phys. 54, 5237 (1971).

[26] P. Tarazona, Mol. Phys. 52, 81 (1984).

[27] V. Talanquer and D. W. Oxtoby, J. Chem. Phys. 103, 3686 (1995).

[28] M. W. Matsen, Phys. Rev. Lett. 74, 4225 (1995).

[29] The present work is in the NVT ensemble, consistent with previous works using real-space SCFT methods without presupposed phases. E. Reister and G. H. Fredrickson have recently shown that this approach is also valid in the grand canonical ensemble; J. Chem. Phys. 123, 214903 (2005).

[30] D. Duchs and D. E. Sullivan, J. Phys.: Condens. Matter 14, 12189 (2002).

[31] Fluctuations mean that no true long-range order can exist in 2D solids, but this aspect has not been accounted for in the present mean-field approach. Following the attitude of Wojciechowski in Ref. [37], this is to avoid complications and because the structures found and the transitions between them are believed to originate mainly from local molecular arrangements.

[32] K. W. Wojciechowski, A. C. Brańka, and D. Frenkel, Physica A 196, 519 (1993), and references therein.

[33] T. A. Cherepanova and A. V. Stekolnikov, Chem. Phys. 154, 41 (1991).

[34] The $\mathrm{N}_{2}$ system has been studied with Monte Carlo simulations in three dimensions. See for example C. Vega, E. P. A. Paras, and P. A. Monson, J. Chem. Phys. 97, 8543 (1992), and references therein.

[35] Here the term "commensurate" refers to a calculation box size that supports the optimal periodic structure of the material, rather than the commensurability of the structure with some substrate, as is commonly meant when discussing adsorbed $\mathrm{N}_{2}$.

[36] This was an unrestricted minimization, which brings into play questions of vacancies in the determined structures that are beyond the scope of the present discussion.

[37] Wojciechowski gives a formula for the maximum packing of a dumbbell system in two dimensions: K. W. Wojciechowski, Phys. Rev. B 46, 26 (1992). The term $\sqrt{3 / 2}$ of his Eq. (1) should be corrected to $\sqrt{3} / 2$. From this formula, the maximum packing density for $d^{*}=0.33$ is $\sim 1.68$. In this work, the phase diagram only goes to 1.4 due to numerical limitations.

[38] J. D. McCoy, S. J. Singer, and D. Chandler, J. Chem. Phys. 87, 4853 (1987).

[39] At high densities, Monte Carlo or molecular dynamics simulations of this system would be expected to take at least two orders of magnitude longer to perform than the present meanfield approach. 